\title{
Cinq approches didactiques pour l'apprentissage d'une langue étrangère à l'ère du numérique
}

\author{
Hussein Taha \\ Faculté d'éducation, Université de Sohag
}

DOI:

\section{Résumé}

A l'ère du numérique où de maintes exigences sont imposées aux acteurs de l'enseignement des langues étrangères, des pédagogues et didacticiens sont à la recherche de nouvelles approches didactiques pouvant aider à une reconfiguration adéquate et efficace du contexte d'apprentissage. Dans ce cadre, le présent article a pour objectif de jeter la lumière sur cinq approches didactiques ayant marqué l'histoire de l'apprentissage d'une langue étrangère. Compte tenu de cet objectif, nous mettons à la disposition des lecteurs une vue générale des connaissances actuelles relatives aux approches en question. Deux éléments d'importance constitueront la base de notre réflexion autour de ces approches: d'abord, les objectifs que les partisans de chaque approche cherchent à atteindre dans une classe de langue et, ensuite, les principes qui devraient respectivement être appliqués dans le contexte d'apprentissage d'une langue étrangère visée.

\section{L'approche communicative}

L'approche dominante de l'enseignement/ apprentissage des langues durant environ 25 ans a été l'approche dite "communicative", ce terme renvoyant à un double niveau: les actes de parole et les situations de communication.

Plusprécisément, l'approche communicative s'est développée en France à partir des années 1970 en réaction contre la moment où l'on remet en cause en Grande-Bretagne l'approche situationnelle et où aux USA la grammaire générative transformationnelle de Chomsky est en plein apogée. selon cette approche, les quatre habiletés peuvent être développées puisque tout dépend des besoins langagiers des apprenants. Il ne suffirait ici pas de connaître les règles grammaticales de la langue étrangère pour communiquer, il faudrait en plus connaître les règles d'emploi de cette langue. L'objectif est d'arriver à une communication efficace.

L'objectif de base de l'approche communicative est de permettre à l'apprenant de construire une compétence de communication ajustée à ses besoins, tels qu'il peut les exprimer ou, le plus souvent, peut les apprendre à sa place. Cet objectif principal entraîne aussi la nécessité de développer chez cet apprenant une capacité à mobiliser ses ressources linguistiques en langue étrangère et ses connaissances de la communication en langue 
maternelle et secondairement en langue étrangère en vue d'une négociation optimale du sens.

La série «En Français Aussi », dont l'objectif primordial est de faire acquérir aux apprenants une compétence globale de communication leur permettant de communiquer oralement et par écrit dans les différents domaines de la vie quotidienne, est divisée en cinq séquences: découvrir le document, observer et déduire, entendre, lire et prononcer, parler et écrire.

L'enseignement de cette série exige que l'enseignant maitrise un ensemble de règles grammaticales qui doivent être utilisées à travers des fonctions communicatives (refus, acceptation, invitation,...). Ces règles enseignées ont pour objectif d'aider les apprenants à bien utiliser la langue française orale et écrite. De même, l'enseignant a pour tâche de perfectionner le contenu linguistique de cette série et qui contient un vocabulaire varié ainsi qu'un grand nombre de situations de valeur communicative. $\mathrm{La}$ présentation orale de ce contenu doit suivre certainement les critères de prononciation reconnus (liaison, enchaînement, articulation, etc.).

\section{- Principes de l'approche communicative}

L'approche communicative regroupe l'ensemble des principes suivants:

- La centration sur l'apprenant: une approche communicative exige un type différent d'organisation de la classe, qui tient compte du fait qu'il s'agit d'un enseignement centré sur l'apprenant et non sur l'enseignant ou sur la méthode ou sur le matériel didactique. Personne comme le constate Meirieu (1991), ne peut se substituer à l'élève dans son apprentissage et ce dernier doit se construire des réseaux entre les concepts et lier les savoirs véhiculés en classe et autour de lui. Cette perspective exige alors que le maitre doive se centrer sur l'élève apprenant afin de modifier son rapport aux savoirs et de favoriser ainsi leur acquisition. Se centrer sur l'apprenant, c'est de s'intéresser à ses processus d'apprentissage, ses modes d'appropriation de la connaissance, ses acquis, l'usage qu'il peut faire de ses connaissances (Martinet et al, 2001). Ainsi, la façon d'enseigner les langues aujourd'hui tend à positionner les deux acteurs enseignant/ apprenant, dans une relation égalitaire. Le savoir se transmet toujours mais surtout le savoir apprendre à apprendre (Tagliante, 1994).

- Savoir une langue, c'est savoir communiquer en connaissant la règle du jeu: il ne suffit pas de savoir le système linguistique, il faut également savoir s'en servir en fonction du contexte social.

- La simulation: elle signifie les tentatives de rapprochement ou d'imitation de situations naturelles. Dans cette approche, les situations d'apprentissage sont plus souvent artificielles qu'authentiques. 
- L'évaluation est au service de l'apprentissage: elle peut être utilisée au processus de construction des savoirs, elle sert au maître et aux apprenants. Elle doit permettre au premier d'être plus efficace en l'aidant à comprendre et à maitriser les situations de classe au quotidien et les difficultés d'apprentissage que les apprenants rencontrent (Léonard, 2000). Egalement, elle soutient les apprenants dans leurs apprentissages dans la mesure où ils sont bien impliqués: on leur demande de se remettre en question, consentir à sortir du rôle passif auquel l'apprentissage scolaire les a habitués et prendre leur apprentissage en main. De coup ils ont droit de regard sur cet apprentissage. Ils définissent leurs propres objectifs et ont le droit de savoir, à tout moment, où ils en sont (Tagliante, 1994).

- Les erreurs d'apprentissage: elles font partie du processus d'apprentissage: considérer l'erreur comme un élément naturel lorsque l'on apprend permettra souvent d'aider à progresser (Hourst, 2004).

- L'équilibre en apprentissage: les quatre compétences langagières sont à pied d'égalité en termes d'enseignement, et leur apprentissage se fait de manière plus équilibrée. Cet équilibre est, inévitablement, une dimension importante à respecter par les enseignants et /ou les formateurs lors la conception des activités d'apprentissage.

\section{L'approche actionnelle / interactionnelle /} fondée sur les tâches

- L'approche fondée sur les tâches part effectivement de l'idée selon laquelle la priorité, dans une tâche d'apprentissage, devait être donnée au sens plutôt qu'à la forme (Guichon, 2006). Pour Nunan (1989, 10), dans son Designing tasks for the communicative class room, une tâche est une unité de travail centrée sur le sens (Meaning-Focused Work) impliquant les apprenants dans la compréhension, la production et / ou l'interaction en langue cible. Egalement, selon Ellis (2003: 5), la tâche est un plan de travail, principalement orienté vers le sens, menant des apprenants à utiliser la langue d'une manière proche de la vie réelle, pouvant concerner une ou plusieurs des quatre compétences, ainsi que des différents processus cognitifs, et possédant un résultat clairement identifiable qui permet de déterminer si la tâche a bien été effectuée. Selon Curran, Williams, Miletto, \& Van der Straeten (2001), l'accent est ainsi placé non pas sur l'apprentissage de points de langage dans un vide non contextualisé, mais sur l'utilisation de la langue en tant qu'outil permettant de satisfaire des besoins authentiques. Dans ce contexte, la langue n'est pas une fin en soi, mais un moyen au service de la tâche à effectuer. 


\section{- L'approche actionnelle et la notion de la tâche}

Selon l'approche actionnelle, la notion de tâche renvoie à une action finalisée, avec un début, un achèvement visé, des conditions d'effectuation, des résultats constatables (Coste, 2009,15). Or, la conception d'une tâche requiert préalablement la prise en compte de six paramètres (Nunan, 1991): les objectifs pédagogiques, les données (ou l'input), l'activité, le rôle de l'enseignant, le rôle des apprenants, le dispositif (settings). D'autres auteurs, relatent Mangenot et Louveau (2006), ont ajouté un septième paramètre: le résultat (l'outcome). Autrement dit, il n'y a tâche que si l'action est motivée par un objectif clairement perçu par les apprenants et si cette action donne lieu à un résultat discernable.

"Est définie comme tâche toute visée actionnelle que l'acteur se représente comme devant parvenir à un résultat donné en fonction d'un problème à résoudre, d'une obligation à remplir, d'un but qu'on s'est fixé (CECRL, 2001, 16)."

La conception d'une tâche requiert préalablement la prise en compte de six paramètres (Nunan, 1991): les objectifs pédagogiques, les données (ou l'input), l'activité, le rôle de l'enseignant, le rôle des apprenants, le dispositif (settings). D'autres auteurs, relatent Mangenot et Louveau (2006), ont ajouté un septième paramètre : le résultat (l'outcome).

Une des caractéristiques de cette tâche est son inscription dans un contexte social donné où les apprenants sont impliqués dans des tâches faisant appel à des pratiques sociales authentiques. Et, ceci donne du sens à l'activité même d'apprentissage.

La perspective privilégiée ici est (...) de type actionnel en ce qu'elle considère avant tout l'usager et l'apprenant d'une langue comme des acteurs sociaux ayant à accomplir des tâches (qui ne sont pas seulement langagières) dans des circonstances et un environnement donnés, à l'intérieur d'un domaine d'action particulier. Si les actes de parole se réalisent dans des activités langagières, celles-ci s'inscrivent ellesmêmes à l'intérieur d'actions en contexte social qui seules leur donnent leur pleine signification (CECRL, 2001, 15).

L'approche/perspective actionnelle requiert que l'apprenant (acteur et usager de la langue), dans une tâche donnée, soit stratégiquement engagé dans un travail de mobilisation des compétences personnelles (linguistiques, discursives, pragmatiques, etc.) en vue d'arriver à réaliser avec succès un produit langagier bien déterminé. Dans ce sens, disent Mangenot \& Penilla (2009, 82), les différentes innovations et expérimentations, auxquelles le CECRL fait écho, partagent une vision pragmatique "actionnelle" de la langue et de son apprentissage, qui s'intéresse à ce que l'apprenant fait et surtout au résultat qu'il atteint en faisant. Les tâches requièrent en effet des participants d'agir principalement en tant qu'utilisateurs de la langue et ainsi d'employer les mêmes processus communicatifs que ceux du monde réel. 
Une autre caractéristique de la tâche présentée dans le CECRL est qu'elle peut être de nature essentiellement langagière ou non langagière.

"La tâche (...) peut être essentiellement langagière, c'est-à-dire que les actions qu'elle requiert sont avant tout des activités langagières et que les stratégies mises en ouvre portent d'abord sur ces activités langagières (par exemple : lire un texte et en faire un commentaire, compléter un exercice à trous, donner une conférence, prendre des notes pendant un exposé). Elle peut comporter une composante langagière, c'est-à-dire que les actions qu'elle requiert ne sont que pour partie des activités langagières et que les stratégies mises en ouvre portent aussi ou avant tout sur autre chose que ces activités (ex. : confectionner un plat à partir de la consultation d'une ficherecette) (CECRL, 2001, 19)."

En suivant Guichon (2006), une tâche d'apprentissage d'une langue, dans une approche par les tâches, devrait avoir un ensemble de caractéristiques qui peuvent être décrites comme suivi:

- Une tâche fournit un cadre à l'activité d'apprentissage. S'engager dans une tâche, c'est avoir un projet, être à même d'anticiper les moyens à mettre en place et pouvoir se représenter le résultat.

- Une tâche implique que la priorité soit donnée au sens. Elle cherche à conduire les apprenants à une utilisation de la langue qui soit pragmatique plutôt que gratuite.
- Une tâche implique que l'utilisation de la langue se fasse selon des critères se rapprochant de la vie extrascolaire et peut inclure toutes les compétences langagières (compréhension, production, interaction).

Une tâche débouche sur un produit langagier clairement défini.

En résumé, quatre éléments apparaissent dans les définitions ci-dessus et constituent ainsi des éléments de similitude entre les deux approches: l'implication de l'apprenant dans la tâche d'apprentissage, la priorité accordée à la construction du sens par rapport à la forme, l'authenticité de la tâche et le traitement de l'information nécessitant un effort croissant de la part de l'apprenant qui mobilise toutes ses connaissances déjà stockées à la mémoire à long terme en fonction des données de la tâche sociale à réaliser.

Cependant, ces définitions laissent une certaine ambiguité à propos des types de tâches d'apprentissage même si elles évoquent la possibilité qu'une tâche peut être langagière ou non langagière. Certains comme Mangenot et Louveau (2006) préfèrent l'usage de la classification (tâche fermée \& tâche ouverte). Une tâche ouverte doit impliquer une production langagière, et ceci fait impérativement appel à une interaction. Tandis qu'une tâche fermée n'est pas conditionnée par une production langagière. D'autres comme NarcyCombes (2005) et Guichon (2006) évoquent une 
autre classification de type "micro- tâches \& macrotâches". La macro-tâche se définit comme un ensemble d'actions constituant une forme de "mise en scène" de la réalité. Elle conduit à une production langagière non limitée à l'univers scolaire, à un projet d'apprentissage global au cours duquel les apprenants sont amenés à traiter de l'information écrite ou orale en langue 2 pour construire un objet de sens écrit ou oral. Une micro-tâche est plus circonscrite et moins réaliste et doit inclure gestion du sens et focalisation de l'attention sur certains aspects précis de la langue.

\section{Approche fondée sur l'économie de la connaissance}

L'économie de la connaissance est un concept récemment apparu sur la scène pédagogique grâce à l'accélération sans précédent de la connaissance et au rôle joué par la technologie dans l'acquisition de cette connaissance. Selon Melbouci et Souki (2014, 4), l'intérêt que lui portent les théoriciens s'est imposé suite aux changements des facteurs qui déterminent la performance et la compétitivité et s'orientent davantage vers l'immatériel.

Or, l'économie de la connaissance est devenue un des vecteurs principaux ayant pénétré dans tous les secteurs d'investissement qui reconnaissent le rôle de la connaissance dans la réalisation $\mathrm{du}$ développement. Certes, le rôle de la connaissance est déjà reconnu par les théoriciens et les économistes, mais le fait qu'elle soit au premier rang des fondements de l'économie est récemment validé.

Dans cette perspective, la richesse des individus et des nations repose actuellement davantage sur la possession de la connaissance et la maîtrise des compétences que sur l'avoir des ressources naturelles. Cette économie ainsi favorise la création et l'utilisation du savoir, la diffusion rapide $\mathrm{du}$ savoir et l'emploi de nouvelles technologies. Ceci pose alors un certain nombre de défis qui touchant tous les secteurs de la vie et y compris éducatif.

Evidemment, le secteur éducatif ne peut être à la marge, loin de ce courant économique et de ce défi majeur qui valorise la recherche et la production de la connaissance. On a ainsi entendu parler de l'économie de l'enseignement, de la société de la connaissance et de la révolution des informations. Ledéfi consisterait, alors, à préparer les apprenants à cette nouvelle société, celle de la connaissance. Il s'agit d'un défi qui change tout, en termes d'apprentissage et d'enseignement et qui impose un certain nombre de mécanismes et de principes organisationnels et pédagogiques qu'il fallait mettre en œuvre dans tous les cycles d'enseignement.

Selon cette approche, deux types de connaissances sont à distinguer: la connaissance déclarative (explicite/ théorique) etla connaissance procédurale (implicite et opérationnelle). Quatre questions sont à poser lors de l'assimilation et de la production de la connaissance: quelle connaissance va-t-on 
acquérir? Pourquoi l'acquérir? A qui on l'attribue? Et, comment l'acquérir?

L'objectif de cette approche est de faire acquérir aux apprenants un ensemble de compétences reliées à la langue et communication, à la pensée critique et créative, à la résolution des problèmes, à la collaboration, au sens de l'initiative, à l'adaptabilité, à la conduite (leadership), et à la conscience technologique, sociale et culturelle. Ces compétences sont ainsi classées en trois catégories: langagières et technologiques, réflexives, et sociales (Ahmed, 2019).

\section{- Principes de l'approche fondée sur}

\section{l'économie de la connaissance}

L'approche fondée sur l'économie de la connaissance regroupe l'ensemble des principes suivants:

- La perspective constructiviste de la connaissance: l'approche fondée sur l'économie de la connaissance favorise une approche constructiviste de l'information. La mise en situation préalable au traitement de l'information, la recherche ou la production d'informations et une collaboration plus ou moins importante entre les apprenants, toutes ces pratiques relèvent du constructivisme.

- Le dynamisme et l'authenticité du contexte d'apprentissage: cette approche crée un contexte d'apprentissage authentique ancré dans la réalité et permet aux apprenants de comprendre en profondeur un thème complexe. Dans ce contexte, l'apprenant est au cœur du processus d'apprentissage et acteur de ses connaissances: il part d'un problème à résoudre, détermine les étapes de sa résolution, recueillit de maintes informations appartenant à plusieurs sources d'apprentissage surtout des sites Web, analyse et synthétise ses idées, interagit avec des coéquipiers, modifie certaines idées (si nécessaire) suite à ces interactions, et présente le fruit de tout ce travail sous forme d'un produit langagier.

- L'intégration des TIC: cette approche repose sur l'intégration des TIC dans l'acte d'apprendre. Or, il est favorablement conseillé de mettre, à la disposition des apprenants, des ressources d'apprentissage variées surtout celles plurimédia et hypertextuelles des nouvelles technologies de l'information et de la communication. L'apprenant peut ainsi trouverune base de documents authentiques, des environnements multimédias interactifs et virtuels d'apprentissage. L'intégration des TIC marque, en fait, un effet positif sur l'engagement, la motivation et l'intérêt des apprenants, et sollicite des opérations cognitives complexes.

- La créativité: les activités d'apprentissage, ainsi que les types de tâches imposées à l'apprenant devraient faire appel à la créativité de celui-ci. Autrement dit, le produit final doit être original 
et répondre aux critères de la créativité surtout l'originalité, la flexibilité cognitive et la fluidité langagière.

\section{L'approche interculturelle}

L'approche interculturelle s'est manifestée dans une période caractérisée par les valeurs de l'individualisme et de la compétitivité (Belkaïd, 2002). Pour faire face à ces situations, cette approche travaille à la prise en considération de la diversité sociale et culturelle des sociétés de pluralisme relevant de ce qu'on a appelé la postmodernité.

L'idée fondatrice de l'approche interculturelle est de s'intéresser à ce qui se passe concrètement lors d'une interaction entre des interlocuteurs appartenant à des communautés culturelles différentes, donc porteurs de schèmes culturels différents, même s'ils communiquent dans la même langue (Serghini, 2014). D'après Blanchet (2004), il s'agit de prévenir, d'identifier, de réguler les malentendus, les difficultés de la communication, dus à des décalages de schèmes interprétatifs, voire à des préjugés (stéréotypes, etc.).

Certes, toute langue véhicule avec elle une culture dont elle est à la fois la productrice et le produit. Conséquemment, les échanges langagiers deviennent des rapports de pouvoir symbolique où s'actualisent les rapports de force entre les locuteurs ou leurs groupes respectifs.
L'importance de l'apprentissage de la culture a un double objectif; d'une part, la valeur de la langue du point de vue socioculturel et sa réussite dans l'acte de communication. D'autre part, la possibilité de diminuer les problèmes envisagés en s'approchant des autres cultures et les dépasser sans aucune difficulté.

On pourrait déduire que chaque nation conserve sa propre identité et parsuite sa propre culture dont elle regroupe les principes, les valeurs, les croyances,les styles de vie et de pensée. L'individu peut exister n'importe où, il peuts'intégrer dans une nouvelle société loin de ses origines mais il gardera tou jourssa propre culture. De plus, au cours de leur vie, les individus acquièrent des comportements; des principes et des normes et des valeurs sociales. En d'autres termes, l'enseignement-apprentissage d'une langue étrangèren'est pas purement linguistique, mais également d'un usage culturel. Cette diversité culturelle et linguistique plante le décor d'un environnement didactique complexe dans lequel enseigner une langue et sa culture est étroitement lié à la construction identitaire des apprenants.

En réalité, le travail autour de cette approche interculturelle se donne pour but de/d' (Byram \& d'autres, 2002):

- Aider les apprenants à comprendre le fonctionnement des interactions interculturelles. 
- Montrer que les identités sociales font partie intégrante de toute relation dans ce domaine.

- Démontrer l'influence de la perception que l'on a des autres et de la vision que les autres ont de vous-même sur la réussite de la communication.

- Amener les apprenants à en savoir plus, par eux-mêmes, au sujet des personnes avec lesquelles ils communiquent.

- Initier l'apprenant à un minimum de connaissances indispensables sur les réalités de la société française: connaissances de type informatif et factuel.

- Inciter l'apprenant à respecter l'altérité culturelle sans pour autant en admettre les principes qui s'apposent à la religion et aux valeurs de l'apprenant.

- Amener l'apprenant à relativiser sa propre culture d'origine ainsi que la culture française.

- Faire acquérir à l'apprenant un savoir- faire interprétatif tout en prenant en considération la différence culturelle entre le natif (l'égyptien) et l'étranger (le français).

- Inciter l'apprenant à établir des comparaisons systématiques entre les questions évoquées dans la culture française et celles qui se posent dans sa culture d'origine.

- Remettre en cause les stéréotypes que l'apprenant a déjà formés sur la France et les français.
- Développer l'esprit critique de l'apprenant, tout en l'amenant à évaluer les aspects de la culture française et ceux de sa propre culture avec tout ce qu'ils comportent d'idées.

\section{- Principes de l'approche interculturelle}

Cinq principes fondamentaux caractérisent l'approche interculturelle (Galisson, 1997, Abdallah-Pretceille, 2004):

- Les représentations et les stéréotypes chez les apprenants: la prise en considération des représentations est considérée comme l'un des fondements principaux de l'approche interculturelle. En effet, elle permet de traiter les aspects socioculturels dans leur hétérogénéité. Ces représentations ne sont plus créées d'une façon impartiale car elles sont simplificatrices et réductrices de la réalité, en autre, il est fixé et se transmit de génération en génération.

- Le dynamism: le terme « dynamisme » représente le lien réciproque qui existe entre des indices de la culture cible et la culture mère. L'objectif de ce lien est d'envisager les divers critères de différences et de ressemblance entre les deux cultures de natures différentes

- La recherche du différent chez l'apprenant:dans le contexte du développement culturel des apprenants de la classe du FLE, l'approche interculturelle expose les divers aspects de la 
culture françaisequi se distinguent de la culture propre des apprenants.

- La recherche du semblable chez l'autre: en plus, la recherche du diffèrent, l'autre s'intéresse aussi à la recherchede tout ce qui est similaire chez l'autre. Or, c'est la faces la moins prospectée et laplus difficile à travailler aussi, parce qu'elle n'occasionne aucun dépaysement, aucun étonnement susceptibles de faire naitre la curiosité, puis la motivation.

- La formation plutôt que l'information: l'approche interculturelle est une approche des cultures basée sur la compréhension plutôt que sur la description.

\section{L'approche pragma-dialectique}

Basée sur une théorie développée par Grootendorst et Van Eemeren, l'approche pragma-dialectique a été proposée à l'Université d'Amsterdam. La pragma dialectique est d'abord une théorie de l'argumentation utilisée pour analyser et évaluer les arguments émis par les acteurs sociaux au sein de leurs pratiques langagières. Ces chercheurs, inspirés par une philosophie critique rationaliste de Karl Popper, ont commencé à étudier l'argumentation comme moyen de résoudre les différences d'opinion. Leur objectif d'étude était alors de développer un code de conduite pour le discours argumentatif.

Les auteurs ont ainsi nommé leur approche de l'argumentation" pragma-dialectique". Cette appellation combine les termes pragmatique et dialectique: pragmatique car elle considère le discours comme un échange d'actes de langage, et dialectique parce qu'elle voit dans cet échange une tentative méthodique de résoudre une différence de points de vue. Il s'agit d'uneprocédure interactionnelle destinée à résoudre un conflit d'opinions au moyen d'un échange réglé d'actes de langage (Van Eemeren\& d'autres, 1996).

Ainsi, l'approche pragma-dialectique considère l'argumentation comme un acte de langage visant à résoudre un conflit d'opinion. Cette approche emploie celle des actes de langage comme outils adéquats pour aborder la communication verbale orientée à résoudre une différence d'opinion.

Selon Van Eemeren, les actes de langage : assertifs, les directifs, les promissifs, les expressifs et les déclarations, peuvent jouer un rôle spécifique à chacune des étapes de la discussion critique (Van Eemeren, Houtlosser \& Snoeck, 2007). Ainsi, en identifiant les actes de langage présents dans chacune des étapes, on peut identifier les profils dialectiques dans un dialogue argumentatif.

Selon Walton (2006) un dialogue est un type de conversation dirigée vers un objectif, à laquelle deux personnes (au moins) participent par des tours de parole; un dialogue argumentatif est une " séquence de mouvements reliés » entre eux (d'actes de langage) dans lesquels les participants posent des questions critiques et y répondent, pour tester la pertinence (à valeur expérimentale, provisoire) 
d'un argument et résoudre ainsi une différence d'opinion.

Dans cette optique, l'argumentation est donc un échange dialogique de mouvements entre deux interlocuteurs ou plus. L'argumentation implique un dialogue parce qu'elle survient toujours en réponse à quelque différence d'opinion, à un doute ou à une critique exprimés ou anticipés (Maingueneau \& d'autres, 2012).

Pour la pragma-dialectique telle qu'elle a été développée par Van Eemeren, l'objectif normatif $\mathrm{du}$ dialogue est la résolution d'une différence d'opinion d'une manière rationnelle, ou selon ses mérites, et l'argumentation est comprise comme une procédure destinée à tester l'acceptabilité d'un point de vue à la lumière d'un questionnement critique (Van Eemeren, 2010).

\section{- Principes de l'approche pragma- dialectique}

L'objet de la théorie pragma-dialectique de l'argumentation est construit en fonction de quatre méta-principes: cet objet est fonctionnel (functionalization), exprimable (externalization), social (socialization) et dialectique (dialectification) (Van Eemeren \& Grootendorst, 2004: 52).

- Fonctionnel: les éléments du discours argumentatifs ne sont pas vus comme des inférences logiques isolées, mais comme des instruments de la communication quotidienne. On ne peut rendre compte du rôle précis de certaines expressions verbales qu'en les considérant comme des actes de langage à part entière dans la situation de communication.

- Exprimable : au lieu de spéculer sur des croyances ou des pensées, on s'appuie sur des énoncés implicites ou explicites. Une reconstruction adéquate permet d'exploiter les indications explicites fournies par le locuteur, mais aussi de dégager certains éléments implicites.

- Social:l'argumentation n'est pas considérée comme un raisonnement produit par un seul locuteur, mais comme une argumentation à laquelle plusieurs locuteurs prennent part.

- Dialectique: l'argumentation est vue comme un moyen rationnel de convaincre un opposant critique, et non comme un moyen de persuasion. Il s'agit de résoudre un conflit en convainquant méthodiquement le locuteur selon des normes critiques de rationalité (dans le cadre de la discussion critique).

Ces postulats jouent un rôle dans l'étude pragmadialectique de l'argumentation: en effet, plusieurs locuteurs (principe social) prennent partà une discussion critique (principe dialectique) ayant pour but de résoudreune divergence d'opinion (principe exprimable) en testant l'acceptabilité oula non acceptabilité (principe fonctionnel) des standpoints (pointsde vue) enjeu dans cette divergence d'opinion. Cela signifie que dans une divergence d'opinion, deux ou plusieurs standpoints sont en 
jeu et le but est derésoudre cette divergence l'opinion. (Miserez-Caperos, 2017:32).

Van Eemeren et Grootendorst ont élaboré le modèle idéal de la discussion critique pour expliquer ce qu'est l'approche pragmadialectiquedans l'usage du langage argumentatif comme un moyen de résoudre des divergences d'opinion. Ce modèle distingue quatre étapes dans le processus de résolution d'une divergence d'opinion de manière argumentative: Confrontation, ouverture, argumentation et conclusion.

Dans la première étape, il devra être évident que l'un des participants n'accepte pas un point de vue particulier et qu'il a des objections; cette différence peut être explicite ou implicite.Dans la deuxième étape, les personnes impliquées décident si elle spartagent des informations suffisantes, des faits ou des données quipermettent de déclencher une discussion fructueuse. Dans cette deuxi èmeétape, l'un des participants assume le rôle de protagoniste, défendant unpoint de vue donné, alors que l'autre répond de manière critique, assumant le rôle d'antagoniste .

Dans l'étape de l'argumentation, le protagoniste propose des arguments qui soutiennent son point de vue et qui contredisent les réponses de l'antagoniste. Dans cette étape, le protagoniste et l'antagoniste peuvent partager quelques points de vue.
Dans l'étape de la conclusion, les parties doivent évaluer le résultat de toute la discussion. La discussion peut être déclarée close uniquement si les parties considèrent que l'argument ou les arguments d'une des parties sont acceptables ou incontestables. C'est ainsi que se termine la discussion critique.

Dans un tel contexte, un principe fondamental qui est à la base de la méthode utilisée par l'approche pragma-dialectique est celui de la reconstruction : Le but d'une analyse pragma-dialectique est de reconstruire le processus de résolution d'une divergence d'opinion survenant dans un discours argumentatif (Van Eemeren \& Grootendorst, 2004: 95).

Cela signifie que la réalité argumentative est, comme le constate Van Eemeren (2004), systématique ment analysée selon la perspective de la discussion critique. Toutes les composantes du discours (ou du texte) qui sont importantes pour la résolution d'une divergence d'opinion sont prises en compte dans une reconstruction; toutes les composantes qui sont sans importance ou sans rapport pour la résolution d'une divergence d'opinion sont laissées de côté.

\section{Références}

Abdallah-Precteille. M., (2004). L'éducation interculturalité. Paris: PUF. 
Ahmed, D., (2019). Les compétences de l'économie du savoir comportées dans les manuels scolaires du secondaire en français langue étrangère et le degré de leur maitrise chez les enseignants. Revue des Sciences pédagogiques, Faculté de Pédagogie de Quéna, 40, 1-28.

Belkaï, M., (2002). La diversité culturelle: pour une formation des enseignants en altérité. Bruxelles: De Boeck Supérieur.

Blanchet, P., (2004). L'approche interculturelle en didactique du FLE, coursd'UED de Didactique du Français Langue Étrangère de 3e année deLicences. Service Universitaire d'Enseignement à Distance, UniversitéRennes 2 Haute Bretagne.

Byram, M. \& Neuner, G., (1992). La compétence interculturelle. Conseild'Europe.

Conseil de 1'Europe., (2001). Un cadre européen commun de référence pour les langues: apprendre, enseigner, évaluer. Paris: Didier.

Curran, P, Deguent, S, Williams, S, Miletto, L.H. \& Van der Straeten, C., (2001).T-Kit: Une méthodologie de l'apprentissage des langues. Strasbourg: Editions du Conseil de l'Europe.

Ellis, R., (2003). Task-based Language Learning and Teaching.Oxford: Oxford University Press.

Galisson, R., (1997). De la langue à la culture par les mots? Paris: Cléinternational.

Guichon, N., (2006): Langues et TICE: Méthodologie de conception Multimedia. Paris: Ophrys.
Hourst, B., (2004). Former sans ennuyer. Paris : Editions d'Organisation.

Léonard, F., (2000). L'évaluation des élèves. In R. La Borderie (Ed). L'école du XXI siècle, pp.75-85. Paris : Nathan.

Maingueneau, D., (1987). Nouvelles tendances en analyse du discours. Paris, Hachette.

Mangenot F. \& Louveau E., (2006). Internet et la classe de langue, Paris: CLE International.

Mangenot, F. \& Penilla, F., (2009) Internet, tâches et vie réelle. Le Français dans le Monde, 45, 82-90.

Martinet, A., Raymond, D. \& Gautier, C., (2001). La formation à l'enseignement. Québec: Ministère de l'Education. Bibliothèque nationale du Québec.

Meirieu, P., (1991). Itinéraire des pédagogies de groupe. Apprendre en groupe. $4 \mathrm{e}$ édition. Paris : Chronique Sociale.

Melbouci, L.\&Souki, H. (2014). Les fondements de l'économie de la connaissance: l'approche basée sur les compétences (ABC) Revue Darassat Iqtissadia, 1: 5-24.

Miserez-Caperos, C., (2017). Etude de l'argumentation à vise cognitive dans des interactions entre adulte et enfants. Doctoral dissertation, Université de Neuchâtel.

Narcy-Combes, J.-P., (2005). Didactique des langues et TIC: vers une recherche-action responsable. Paris: Ophrys. 
Nunan, D., (1989). Designing Tasks for the Communicative Classroom.Cambridge: Cambridge University Press.

Serghini, J., (2014). Pour une approche interculturelle du texte littéraire à traversles textes des écrivains maghrébins et subsahariens de la nouvelle génération. Université Mohammmed premier.

Tagliante, C., (1994). La classe de langue. Paris: CLE international.

Van Emeren, F. H., (2010).Strategic Manoeuvring in Argumentative Discourse. Amsterdam: John Benjamin's.
Van Emeren, F. H. \& Houtlosser, P., (2003). «The Development of the Pragma-Dialectical Approach to Argumentation. Argumentation, 17: 387-403.

Van Emeren, F. H \& Grootendorst, R., (2004). A Systematic Theory of Argumentation: The PragmaDialectical Approach. Cambridge: Cambridge University Press.

Van Emeren, F. H., Houtlosser, P., \& Snoeck, F., (2007). Argumentative indicators in discourse: A pragma dialectical study.Dordrecht, The Netherlands: Springer.

Walton, D., (2006). Fundamentals of Critical Argumentation (New York: Cambridge University Press. 